\title{
Multicritical Point on the de Almeida-Thouless Line in Spin Glasses in $d>6$ Dimensions
}

DOI:

10.1103/PhysRevLett.120.130602

\section{Document Version}

Final published version

Link to publication record in Manchester Research Explorer

\section{Citation for published version (APA):}

Moore, M. A., \& Read, N. (2018). Multicritical Point on the de Almeida-Thouless Line in Spin Glasses in d>6 Dimensions. Physical Review Letters, 120(13), [130602]. https://doi.org/10.1103/PhysRevLett.120.130602

\section{Published in:}

Physical Review Letters

\section{Citing this paper}

Please note that where the full-text provided on Manchester Research Explorer is the Author Accepted Manuscript or Proof version this may differ from the final Published version. If citing, it is advised that you check and use the publisher's definitive version.

\section{General rights}

Copyright and moral rights for the publications made accessible in the Research Explorer are retained by the authors and/or other copyright owners and it is a condition of accessing publications that users recognise and abide by the legal requirements associated with these rights.

\section{Takedown policy}

If you believe that this document breaches copyright please refer to the University of Manchester's Takedown Procedures [http://man.ac.uk/04Y6Bo] or contact uml.scholarlycommunications@manchester.ac.uk providing relevant details, so we can investigate your claim.

\section{OPEN ACCESS}




\title{
Multicritical Point on the de Almeida-Thouless Line in Spin Glasses in $d>6$ Dimensions
}

\author{
M. A. Moore ${ }^{1}$ and N. Read ${ }^{2}$ \\ ${ }^{1}$ School of Physics and Astronomy, University of Manchester, Manchester M13 9PL, United Kingdom \\ ${ }^{2}$ Department of Physics, Yale University, P.O. Box 208120, New Haven, Connecticut 06520-8120, USA
}

(Received 28 January 2018; published 28 March 2018)

\begin{abstract}
The de Almeida-Thouless (AT) line in Ising spin glasses is the phase boundary in the temperature $T$ and magnetic field $h$ plane below which replica symmetry is broken. Using perturbative renormalization group (RG) methods, we show that, when the dimension $d$ of space is just above six, there is a multicritical point (MCP) on the AT line, which separates a low-field regime, in which the critical exponents have mean-field values, from a high-field regime, where the RG flows run away to infinite coupling strength; as $d$ approaches six from above, the MCP approaches the zero-field critical point exponentially in $1 /(d-6)$. Thus, on the AT line, perturbation theory for the critical properties breaks down at a sufficiently large magnetic field even above 6 dimensions, as well as for all nonzero fields when $d \leq 6$, as was known previously. We calculate the exponents at the MCP to first order in $\varepsilon=d-6>0$. The fate of the MCP as $d$ increases from just above six to infinity is not known.
\end{abstract}

DOI: 10.1103/PhysRevLett.120.130602

The nature of the ordered phase of spin glasses has been controversial for decades. When various standard calculational methods are applied to it, the results are sometimes in conflict. The picture that derives from mean-field theory (valid at least for infinite-dimensional systems) is that of replica symmetry breaking (RSB) [1-5]. However, the results of real-space renormalization group (RG) calculations favor an ordered phase with replica symmetry when the dimension $d$ of space is small [6-11]. Recent calculations using the strong-disorder renormalization group were interpreted as suggesting that the spin glass (SG) phase is replica symmetric for $d \leq 6[7,8]$. Much of the debate on the existence or not of RSB has focused on the de Almeida-Thouless (AT) line [12]. According to the RSB theory, there is a phase transition in an applied magnetic field $h$, occurring along the AT line $T_{c}(h)$ as the temperature $T$ is reduced. Below $T_{c}(h)$, there is the SG phase with RSB, whereas for $T \geq T_{c}(h)$, replica symmetry is unbroken. The existence of the AT line in high dimensions $d \geq 6$ is supported by, for example, Ref. [13]. The existence of such a line in three dimensions has been the subject of experimental work [14] and controversial simulational studies [15-18].

In early work, Bray and Roberts (BR) [19] derived a "reduced" field theory of Landau-Ginzburg-Wilson type for a set of fluctuating fields that remain critical on the AT line. Applying standard perturbative RG methods at oneloop (i.e., lowest nontrivial) order, they showed that, when $d$ is less than or equal to 6 , the coupling constants run away to infinity, so no stable physical RG fixed point exists, and hence corrections to the mean-field exponents could not be calculated even at leading order in $6-d$. (This is in contrast with the transition at $h=0$, for which such an expansion exists in the conventional way [20], using the unreduced theory.) BR suggested that, for $d<6$, the transition on the AT line could become first order or the line itself could disappear. When $d>6$, the BR RG flows have a domain of attraction of the zero coupling fixed point [21], so that sufficiently small initial values of the couplings run toward zero, implying mean-field values for critical exponents, while initial values outside this domain run off to infinity; this domain shrinks to zero size as $d \rightarrow 6^{+}$. It is also known that the form of the AT line at small $h$ is modified from the mean-field result for dimensions $6<d<8$ [22,23].

In this Letter, we approach the problem from the point of view of dimensions $d$ larger than 6 . We calculate the crossover from the unreduced to the reduced theory using perturbative RG methods at one-loop order. We find that for sufficiently small $h$, the initial values of the couplings in the BR theory lie inside the domain of attraction of zero coupling, but as $h$ increases, they pass through the boundary of the domain and so run off to infinity. Hence, there is a transition associated with an RG fixed point on the boundary of the domain of attraction. This implies that there is a multicritical point (MCP) $M$ at $(T, h)=\left(T_{M}, h_{M}\right)$ on the AT line for $d>6$, at least for $d$ not much larger than 6 . For small fields, the critical behavior is that of mean-field theory, while for larger fields it is some other unknown behavior (possibly first order); see Fig. 1. The distance in temperature of the MCP from the $h=0$ critical point varies as $c^{1 / \varepsilon} \rightarrow 0$ as $\varepsilon=d-6 \rightarrow 0^{+}$, where $c$ is some constant $(0<c<1)$. We calculate the exponents at the MCP at first order in $\varepsilon$.

As a consequence, non-mean-field behavior of the critical properties occurs on a portion of the AT line already for $d>6$. Its existence suggests the possibility of similar behavior for $d \leq 6$ as well, in this case for all 


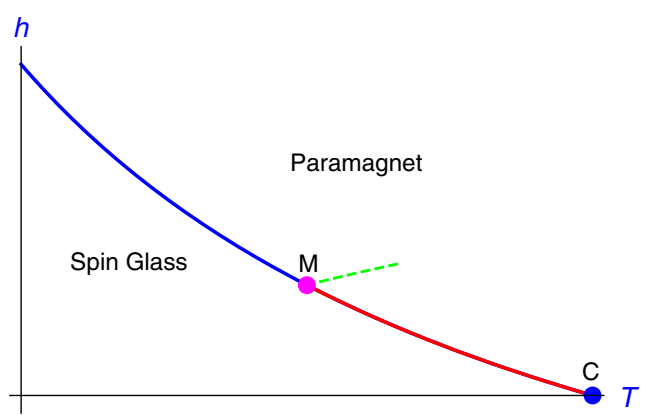

FIG. 1. A schematic phase diagram, for dimension $d$ slightly larger than six, showing the MCP $M$ on the AT line, at which the nature of the criticality changes. The portion of the AT line at low $h$ (red) is where the exponents at the line are mean-field-like; the portion of the line at high $h$ (blue) is where the RG flows run away to infinity. The dashed line (green) indicates schematically the direction along which the distinct correlation length exponent of the multicritical fixed point might be observable. $M$ approaches the zero-field critical point $C$ as $d$ tends to six from above.

$h \neq 0$. However, the possibility that the entire (non-meanfield-like) AT line disappears at once for $d \leq 6$ cannot be excluded using the present methods. Clearly, it is imperative to understand the nature of the non-mean-field part of the AT line. The AT line is expected to intersect the $T=0$ axis at $h=h_{c}$ when $d$ is finite. As $d \rightarrow \infty$ at fixed $T$ and $h$, one expects that mean-field theory becomes exact for the phase boundary and exponents, and hence, that both $h_{c}$ and $h_{M}$ should tend to infinity as $d$ increases; $M$ will reach $T=0$ either at some finite $d=d_{u}>6$, so there is no nonmean-field portion for $d>d_{u}$, or at $d_{u}=\infty$.

We start from the Edwards-Anderson (EA) model [24] defined on a $d$-dimensional hypercubic lattice of linear extent $L$ by the Hamiltonian

$$
H=-\sum_{\langle i j\rangle} J_{i j} S_{i} S_{j}-h \sum_{i} S_{i},
$$

where the summation is over distinct nearest-neighbor pairs only, the Ising spins take the values $S_{i} \in\{ \pm 1\}$ with $i=1,2, \ldots, L^{d}$, and the random bonds $J_{i j}$ are independent Gaussian variables of variance $(2 d)^{-1 / 2}$ [chosen so that $T_{c}(h=0) \rightarrow 1$ as $d \rightarrow \infty$ ] and zero mean. From the partition function associated with Eq. (1), one can derive $[20,25,26]$ the replicated and bond-averaged LandauGinzburg-Wilson field theory, which involves fluctuating fields $Q_{\alpha \beta}=Q_{\beta \alpha}$, where, as usual, the indices $\alpha$ and $\beta$ run over values $1,2, \ldots, n, n$ is set zero at the end of the calculation, and $Q_{\alpha \alpha}=0$ for all $\alpha$. The action in this theory is [19]

$$
\begin{aligned}
F\left[\left\{Q_{\alpha \beta}\right\}\right]= & \int d^{d} x\left(-\frac{1}{4} r \sum Q_{\alpha \beta}^{2}+\frac{1}{4} \sum\left(\nabla Q_{\alpha \beta}\right)^{2}\right. \\
& -\frac{1}{6} w \sum Q_{\alpha \beta} Q_{\beta \gamma} Q_{\gamma \alpha}-\frac{1}{8} y \sum Q_{\alpha \beta}^{4} \\
& \left.-\frac{1}{2} h^{2} \sum Q_{\alpha \beta}+\cdots\right) .
\end{aligned}
$$

Here the summations in each term are over all values of the free indices in that term and are unrestricted except that $Q_{\alpha \alpha}=0$. Terms omitted are other less-important terms of order $Q^{4}$ or higher or with more than two derivatives. The coefficients $w$ and $y$ are positive, while we have reversed the usual sign of $r$, so that $r \propto T_{c}(h=0)-T>0$ for $T<T_{c}(h=0)$. This theory, to which we refer as the unreduced theory, is usually believed to capture the essence of SG behavior near criticality in $d$ dimensions.

The unreduced theory contains $\frac{1}{2} n(n-1)$ modes when expanded to quadratic order, which can be classified [12] into symmetry types, conventionally called longitudinal (one mode), anomalous ( $n-1$ modes), and replicon $\left[\frac{1}{2} n(n-3)\right.$ modes]. By a standard RG method, in which a cutoff of 1 is assumed, and Fourier components of fields with wave vectors in a shell just below the cutoff are successively integrated out, followed by rescaling to restore the cutoff to 1 , one obtains the one-loop RG flow equations $[20,23,27]$ for the effective couplings $w(l), r(l), h(l)^{2}$, and $y(l)$ at length scale $e^{l}$ (where scale $l=0$ corresponds to the initial cutoff scale),

$$
\begin{gathered}
\frac{d w}{d l}=\frac{1}{2}[-\varepsilon-3 \eta] w-2 w^{3}, \\
\frac{d r}{d l}=\left[2-\eta-4 w^{2}\right] r, \\
\frac{d h^{2}}{d l}=\frac{1}{2}[d+2-\eta] h^{2}, \\
\frac{d y}{d l}=\left[4-d-2 \eta-B w^{2}\right] y+A w^{4},
\end{gathered}
$$

where $\varepsilon=d-6, \eta=-\frac{2}{3} w^{2}$, and $A>0$ and $B$ are constants, the values of which are not important. We adopted the convention of absorbing the geometric factor $K_{d}=$ $2 /\left[\Gamma(d / 2)(4 \pi)^{d / 2}\right]$ into $w^{2}$. Mass corrections in denominators in these equations [27] have been dropped, except in the RG equation for $r$, where the first order term has been retained. (That equation should also include an inhomogeneous term that describes a shift in the critical temperature; however, that effect is also negligible in the limit we consider.)

The flow equations can be solved exactly. First, one has [21]

$$
w(l)=\frac{w_{0} e^{-\frac{1}{2} \varepsilon l}}{\left[1+\frac{2 w_{0}^{2}}{\varepsilon}\left(1-e^{-\varepsilon l}\right)\right]^{1 / 2}},
$$

where $w_{0}=w(0)$, which typically is of order 1 . We will see that the matching to the $\mathrm{BR}$ reduced action that we require occurs at $w \propto \sqrt{\varepsilon}$ as $\varepsilon \rightarrow 0^{+}$(i.e., $d \rightarrow 6^{+}$), so that the limit of interest in the following is always $\varepsilon \rightarrow 0$ with $\varepsilon l$ (and $w_{0}$ ) fixed. In this limit, 


$$
w(l)=\left(\frac{\varepsilon}{2}\right)^{1 / 2} \frac{e^{-\frac{1}{2} \varepsilon l}}{\left(1-e^{-\varepsilon l}\right)^{1 / 2}}[1+\mathcal{O}(\varepsilon)] .
$$

[Recall that $f=\mathcal{O}(g)$ as $\varepsilon \rightarrow 0$ means that $|f(\varepsilon) / g(\varepsilon)|$ is bounded above for all $\varepsilon$ sufficiently close to 0 .] Similarly [21],

$$
\begin{gathered}
r(l)=r(0) \exp \left[2 l-\frac{10}{3} \Delta(l)\right], \\
h(l)^{2}=h(0)^{2} \exp \left[\frac{1}{2}(d+2) l+\frac{1}{3} \Delta(l)\right],
\end{gathered}
$$

where

$$
\begin{aligned}
\Delta(l) & =\int_{0}^{l} w\left(l^{\prime}\right)^{2} d l^{\prime} \\
& =\frac{1}{2} \ln \left[1+\frac{2 w_{0}^{2}}{\varepsilon}\left(1-e^{-\varepsilon l}\right)\right] \\
& =\frac{1}{2} \ln \left[\frac{2 w_{0}^{2}}{\varepsilon}\left(1-e^{-\varepsilon l}\right)\right]+\mathcal{O}(\varepsilon)
\end{aligned}
$$

in the required limit. For $y$, we obtain likewise

$$
\begin{aligned}
y(l)= & y(0) \exp \left[(4-d) l+\left(\frac{4}{3}-B\right) \Delta(l)\right] \\
& +A e^{(4-d) l+\left(\frac{4}{3}-B\right) \Delta(l)} \int_{0}^{l} w\left(l^{\prime}\right)^{4} e^{-(4-d) l^{\prime}-\left(\frac{4}{3}-B\right) \Delta\left(l^{\prime}\right)} d l^{\prime} .
\end{aligned}
$$

In this case, the required limit can be obtained by defining the integration variable $l^{\prime \prime}=\varepsilon l^{\prime}$, in terms of which the integration limit becomes a constant and Laplace's method can be applied to the integral, to obtain

$$
y(l)=A \frac{\varepsilon^{2} e^{-2 \varepsilon l}}{8\left(1-e^{-\varepsilon l}\right)^{2}}[1+\mathcal{O}(\varepsilon)],
$$

as $\varepsilon \rightarrow 0$ with $\varepsilon l>0$ fixed; the initial value $y(0)$ is an exponentially small correction and has been dropped. Thus, $y=\frac{1}{2} A w^{4}$ [23].

The crossover to the $\mathrm{BR}$ reduced action takes place at the scale $l=l^{*}$, at which the longitudinal and anomalous modes have mass-squared 1 , while by definition of the AT line, the replicons remain massless there. The action (2) predicts at mean-field level that, for nonzero $h^{2}$, the AT line in the $r-h$ plane and the replica symmetric expectation $Q$ of $Q_{\alpha \beta}$ on the line are given by

$$
Q=\frac{r}{2 w}>0, \quad h^{2}=2 y Q^{3}=\frac{y r^{3}}{4 w^{3}} .
$$

These expressions [19] are valid up to corrections of relative size $y r / w^{2}$. Further, the longitudinal and anomalous modes can be shown [12] to have mass-squared $r$ on the AT line. Setting $r\left(l^{*}\right)=1$, the corrections to the leading expressions for $Q$ and $h^{2}$ on the AT line are of relative order $y\left(l^{*}\right) r\left(l^{*}\right) / w\left(l^{*}\right)^{2}=\frac{1}{2} A w\left(l^{*}\right)^{2}=\mathcal{O}(\varepsilon)$ as $\varepsilon \rightarrow 0$ with $\varepsilon l^{*}$ fixed.

The BR reduced action results from (2) by setting the fluctuations of the nonreplicon modes to zero; the fields $\tilde{Q}_{\alpha \beta}$ in the replicon sector are defined by the condition $\sum_{\beta} \tilde{Q}_{\alpha \beta}=$ 0 for all $\alpha$, in addition to $\tilde{Q}_{\alpha \alpha}=0$. The reduced action is [19]

$$
\begin{aligned}
F\left[\left\{\tilde{Q}_{\alpha \beta}\right\}\right]= & \int d^{d} x\left(\frac{1}{4} \tilde{r} \sum \tilde{Q}_{\alpha \beta}^{2}+\frac{1}{4} \sum\left(\nabla \tilde{Q}_{\alpha \beta}\right)^{2}\right. \\
& \left.-\frac{1}{6} w_{1} \sum \tilde{Q}_{\alpha \beta} \tilde{Q}_{\beta \gamma} \tilde{Q}_{\gamma \alpha}-\frac{1}{6} w_{2} \sum \tilde{Q}_{\alpha \beta}^{3}\right),
\end{aligned}
$$

up to terms higher order in $\tilde{Q}$ or derivatives. Here again the summations are unrestricted, but the fields obey the conditions noted above. $\tilde{r}=-r+2 w Q$ [19] vanishes on the AT line. In principle, the nonreplicon modes should be integrated out exactly once they become massive $[r(l) \geq 1]$, not just projected to zero, but this should produce, at most, only negligible $[\mathcal{O}(\varepsilon)]$ corrections to coefficients, because (as we will see) the couplings are of order $\varepsilon^{1 / 2}$.

The remaining coupling constants in the BR reduced action are

$$
\begin{gathered}
w_{1}=w-3 u Q, \\
w_{2}=3 y Q .
\end{gathered}
$$

Here $u$ is another quartic coupling in the unreduced action, for which the flow is the same as for $y$ except that $A$ and $B$ are replaced by some $A^{\prime}$ and $B^{\prime}$. By the above, when the crossover to the BR reduced action occurs, these are

$$
\begin{aligned}
& w_{1}=w, \\
& w_{2}=0,
\end{aligned}
$$

plus terms of order $\mathcal{O}(\varepsilon w)$ in the required limit.

When $w_{2}=0$, the critical $w_{1}$ on the boundary of the domain of attraction of the origin in the BR RG flows (which we review below) is $w_{1}=\left(c^{\prime} \varepsilon\right)^{1 / 2}$, where $c^{\prime}=1 / 24$. Setting $w\left(l_{M}^{*}\right)=\left(c^{\prime} \varepsilon\right)^{1 / 2}$ gives, for $l^{*}=l_{M}^{*}$ at $M$,

$$
\frac{e^{-\varepsilon l_{M}^{*}}}{1-e^{-\varepsilon l_{M}^{*}}}=2 c^{\prime}
$$

that is,

$$
e^{-\varepsilon l_{M}^{*}}=\frac{2 c^{\prime}}{1+2 c^{\prime}}<1 .
$$

Thus, $\varepsilon l_{M}^{*}>0$ is a constant and $l_{M}^{*}$ is large as $\varepsilon \rightarrow 0$, regardless of the precise value of $c^{\prime}$ (note that $c^{\prime}>0$ ).

Using the expressions for $r\left(l^{*}\right)=1$ and $h\left(l^{*}\right)^{2}$, we find the location of the MCP $M$ in terms of the bare (i.e., lattice scale, $l=0$ ) parameters in the unreduced action 


$$
\begin{gathered}
r(0)_{M}=\varepsilon^{-5 / 3}\left(e^{-\varepsilon l_{M}^{*}}\right)^{2 / \varepsilon}\left[2 w _ { 0 } ^ { 2 } \left(1-e^{\left.\left.-\varepsilon l_{M}^{*}\right)\right]^{5 / 3},}\right.\right. \\
h(0)_{M}^{2}=\frac{A \varepsilon^{4} e^{-\varepsilon l_{M}^{*}} r(0)_{M}^{2} w_{0}}{8\left[2 w_{0}^{2}\left(1-e^{-\varepsilon l_{M}^{*}}\right)\right]^{4}},
\end{gathered}
$$

in the limit as $\varepsilon \rightarrow 0$, where $\varepsilon l_{M}^{*}$ is constant. These are among the main results of this Letter; they show that the MCP $M$ approaches the critical point $C$ exponentially fast as $\varepsilon \rightarrow 0$. The exponent in $\varepsilon^{-5 / 3}$ in the first formula should be universal. In the second formula, the fact that $h(0) \propto$ $r(0)$ as $d \rightarrow 6$ (neglecting the prefactor) agrees with Refs. [22,23] (it was derived in a similar way in Ref. [23]), while the $\varepsilon^{4}$ in the coefficient agrees with the results of Ref. [21]; note, however, that the results of these references were valid in the different limit $r(0) \rightarrow 0$ at fixed $\varepsilon$, followed by the $\varepsilon \rightarrow 0$ limit [28].

Next, we turn to calculations that make greater use of the $B R$ reduced theory. BR obtained the one-loop RG equations $[19,27]$

$$
\begin{aligned}
\frac{d w_{1}}{d l}=\frac{1}{2}[-\varepsilon-3 \tilde{\eta}] w_{1}+14 w_{1}^{3}-36 w_{1}^{2} w_{2} \\
+18 w_{1} w_{2}^{2}+w_{2}^{3}, \\
\frac{d w_{2}}{d l}=\frac{1}{2}[-\varepsilon-3 \tilde{\eta}] w_{2}+24 w_{1}^{2} w_{2} \\
-60 w_{1} w_{2}^{2}+34 w_{2}^{3}, \\
\frac{d \tilde{r}}{d l}=[2-\tilde{\eta}] \tilde{r}-\frac{3 \tilde{\eta}}{(1+\tilde{r})^{2}},
\end{aligned}
$$

where now $\tilde{\eta}=\left(4 w_{1}^{2}-16 w_{1} w_{2}+11 w_{2}^{2}\right) / 3$.

Equations (26) and (27) were solved numerically for $d>6$ in Ref. [21]. In the $w_{1}-w_{2}$ plane, the Gaussian fixed point $G$ at $w_{1}=w_{2}=0$ is stable for $d>6 . G$ is the attractor for flows inside the domain of attraction, as shown in Fig. 2. The boundary of the domain is itself a flow line of the RG: a separatrix. There are two pairs of other fixed points on the separatrix [19]: the fixed points $U$ and $-U$ at $w_{1}= \pm \sqrt{\varepsilon / 24}$,

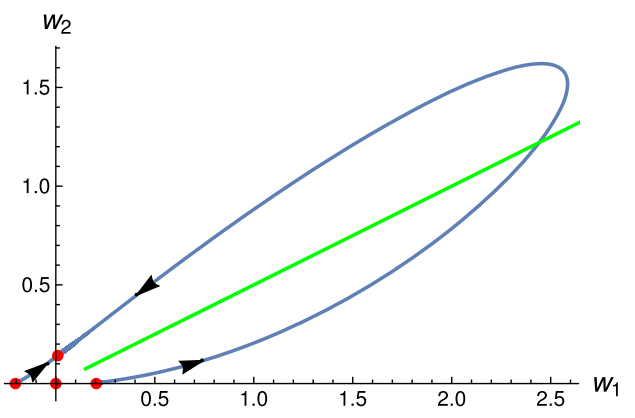

FIG. 2. The domain of attraction of the Gaussian fixed point $w_{1}=w_{2}=0$ of the BR RG flow equations for $d>6$ is bounded by the separatrix shown (blue curve). Distances are measured in units of $\sqrt{\epsilon}$. Only the region $w_{2}>0$ is displayed; the other part is obtained by inversion symmetry. RG fixed points are shown as dots (red). The straight line (green) indicates the initial values. $w_{2}=0$, which are unstable, and the fixed points $Z$ and $-Z$ at $\left(w_{1}, w_{2}\right)= \pm \sqrt{\varepsilon}(0.00983702,0.141449)$, which have one stable (incoming) direction along the separatrix and one unstable direction (marked in orange in Fig. 3). Outside the separatrix, all flows go to infinity.

The exponents for the MCP can be obtained by standard methods from the RG equations linearized at a fixed point. Although we found above that the initial values for the BR flows cross the separatrix at a point approaching $w_{2}=0$ as $\varepsilon \rightarrow 0$, the generic case for $\varepsilon>0$ does not pass through that point, and consequently, the fixed point that controls the true asymptotics of the MCP is $Z$ (see Fig. 3). First, the exponent $\eta$ that describes the power law decay of the replicon correlation function on the AT line,

$$
\overline{\left(\left\langle S_{i} S_{j}\right\rangle-\left\langle S_{i}\right\rangle\left\langle S_{j}\right\rangle\right)^{2}} \sim \frac{1}{r_{i j}^{d-2+\eta}}
$$

(where the overline represents the average over the $J_{i j}$ ), can be found by evaluating $\tilde{\eta}$ at $Z$, giving $\eta=0.06607 \varepsilon$ at $M$. Next, the BR RG equations for $w_{1}$ and $w_{2}$, when linearized about $Z$, produce the eigenvalues $\varepsilon$ for the unstable direction, and $-0.25624 \cdots \varepsilon$ for the stable direction along the separatrix. The first of these describes the crossover as the system is perturbed off the MCP but staying on the AT line, flowing to $G$ if $h^{2}$ is decreased. (The second gives corrections to scaling.) Finally, by linearizing Eq. (28) about the fixed point value of $\tilde{r}$, one can calculate the exponent $\nu$ for the correlation length as the AT line is approached; it is given by $1 / \nu=2+5 \tilde{\eta}$, so at $Z$ (i.e., $M$ ), $\nu=1 / 2-0.082585 \varepsilon$. Hyperscaling relations among exponents are satisfied at $M$, even though $d>6$. The mean-field portion of the AT line at low $h$ is governed instead by $G$, with $\eta=0$ and $\nu=1 / 2$; hyperscaling is violated for $d>6$.

We emphasize that our results are well controlled within perturbation theory, similar to the usual $\varepsilon$ expansion. They predict a breakdown of perturbation theory on the AT line above a critical $h$ when $d>6$. Hence, the only way they could fail to be correct, or the MCP not exist, would be if perturbation theory broke down at all $h>0$ when $d$ is just

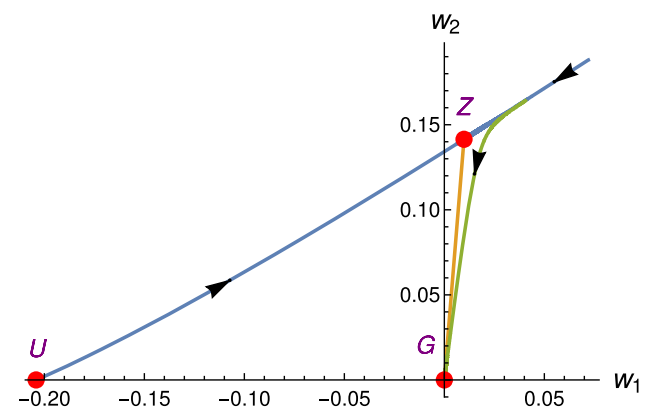

FIG. 3. An enlarged version of Fig. 2 in the vicinity of the origin. The orange line connects the fixed point $Z$ to the Gaussian fixed point $G$ at the origin. The green curve is a trajectory for an initial point just off the separatrix. 
larger than 6; it is unclear how that would occur. Similar methods show that the one-dimensional power law model [29] possesses a MCP in the region corresponding to $d>6$.

It is not clear what happens to the MCP as $d$ increases further. The transition point $h=h_{c}$ at $T=0$ is expected to go to infinity as $d \rightarrow \infty$. In finite dimensions, the BethePeierls approximation, that is, the solution of the SG on the Bethe lattice [30,31], predicts a transition at $T_{c}(h) \rightarrow 0$ at nonzero $h=h_{c}<\infty$, and $h_{c}^{2} \sim \ln d \rightarrow \infty$ as $d \rightarrow \infty$ [32]. (For Gaussian random fields with variance $h^{2}$ and mean zero, $h_{c} \sim \sqrt{2 d}$ instead.) This limit thus agrees with the solution of the Sherrington-Kirkpatrick model [12]. On the Bethe lattice, the $T=0$ transition is percolative in nature [33], but it is not clear if that is true for the EA model at high $d$. No MCP has been found in these other models, so we expect $T_{M} \rightarrow 0$ as $d \rightarrow \infty$. Hence, we define a dimension $d_{u}>6$ at which the MCP hits the $T=0 h$ axis; it is possible that $d_{u}=\infty$.

Recent work has suggested alternative pictures. Reference [9] finds a non-mean-field transition governed by a zero-temperature fixed point at nonzero $h$ for sufficiently high dimensions and no MCP (see also Ref. [34]). However, the exponents for that transition given there for hierarchical-lattice models imply that the SG susceptibility exponent $\gamma$ is negative, which means the SG susceptibility does not diverge at the AT line, at variance with the conventional view of the line. In those models, any finite region is contained in a region with only two spins on the boundary, so there can be at most four ground states. This and a similar limitation on the number of pure states at $T>0$ preclude most forms of RSB a priori (see also Ref. [35]). In other work, Ref. [36] has extended the BR calculation to three-loop order and suggested that a fixed point might be present at strong coupling in 5 dimensions and below, even if not right up to 6 dimensions. Their argument is of unknown validity.

In conclusion, we have shown that there is a nonperturbative (non-mean-field) portion of the AT line in a spin glass in dimensions greater than 6 , separated from the mean-field region at a low magnetic field by a multicritical point. This suggests, though it does not prove, that a similar nonperturbative AT line could also persist below 6 dimensions.

M. A. M. would like to thank Maria Chiara Angelini, Giulio Biroli, Gilles Tarjus, Tamas Temesvári, and Sho Yaida for email discussions and Mike Godfrey for his help with Mathematica.

[1] G. Parisi, Infinite Number of Order Parameters for SpinGlasses, Phys. Rev. Lett. 43, 1754 (1979).

[2] G. Parisi, Order Parameter for Spin-Glasses, Phys. Rev. Lett. 50, 1946 (1983).

[3] R. Rammal, G. Toulouse, and M. A. Virasoro, Ultrametricity for physicists, Rev. Mod. Phys. 58, 765 (1986).
[4] M. Mézard, G. Parisi, and M. A. Virasoro, Spin Glass Theory and Beyond (World Scientific, Singapore, 1987).

[5] G. Parisi, Some considerations of finite dimensional spin glasses, J. Phys. A 41, 324002 (2008).

[6] M. A. Moore, H. Bokil, and B. Drossel, Evidence for the Droplet Picture of Spin Glasses, Phys. Rev. Lett. 81, 4252 (1998).

[7] W. Wang, M. A. Moore, and H. G. Katzgraber, Fractal Dimension of Interfaces in Edwards-Anderson and LongRange Ising Spin Glasses: Determining the Applicability of Different Theoretical Descriptions, Phys. Rev. Lett. 119, 100602 (2017).

[8] W. Wang, M. A. Moore, and H. G. Katzgraber, Fractal dimension of interfaces in Edwards-Anderson spin glasses for up to six space dimensions, Phys. Rev. E 97, 032104 (2018).

[9] M. C. Angelini and G. Biroli, Spin Glass in a Field: A New Zero-Temperature Fixed Point in Finite Dimensions, Phys. Rev. Lett. 114, 095701 (2015).

[10] M. C. Angelini and G. Biroli, Real space renormalization group of disordered models of glasses, Proc. Natl. Acad. Sci. U.S.A. 114, 3328 (2017).

[11] M. C. Angelini and G. Biroli, Real space Migdal-Kadanoff renormalisation of glassy systems: Recent results and a critical assessment, J. Stat. Phys. 167, 476 (2017).

[12] J. R. L. de Almeida and D. J. Thouless, Stability of the Sherrington-Kirkpatrick solution of a spin glass model, J. Phys. A 11, 983 (1978).

[13] R. R. P. Singh and A.P. Young, de Almeida-Thouless instability in short-range Ising spin glasses, Phys. Rev. E 96, 012127 (2017).

[14] J. Mattsson, T. Jonsson, P. Nordblad, H. Aruga Katori, and A. Ito, No Phase Transition in a Magnetic Field in the Ising Spin Glass $\mathrm{Fe}_{0.5} \mathrm{Mn}_{0.5} \mathrm{TiO}_{3}$, Phys. Rev. Lett. 74, 4305 (1995)

[15] T. Jörg, H. G. Katzgraber, and F. Krzakala, Behavior of Ising Spin Glasses in a Magnetic Field, Phys. Rev. Lett. 100, 197202 (2008).

[16] R. A. Baños et al., Thermodynamic glass transition in a spin glass without time-reversal symmetry, Proc. Natl. Acad. Sci. U.S.A. 109, 6452 (2012).

[17] M. Baity-Jesi et al., Dynamical transition in the $D=3$ Edwards-Anderson spin glass in an external magnetic field, Phys. Rev. E 89, 032140 (2014).

[18] M. Baity-Jesi (Janus Collaboration), The three-dimensional Ising spin glass in a field: The role of the silent majority, J. Stat. Mech. (2014) P05014.

[19] A. J. Bray and S. A. Roberts, Renormalisation-group approach to the spin glass transition in finite magnetic fields, J. Phys. C 13, 5405 (1980).

[20] A. B. Harris, T. C. Lubensky, and J.-H. Chen, Critical Properties of Spin Glasses, Phys. Rev. Lett. 36, 415 (1976).

[21] M. A. Moore and A.J. Bray, Disappearance of the de Almeida-Thouless line in six dimensions, Phys. Rev. B 83, 224408 (2011).

[22] J. E. Green, M. A. Moore, and A. J. Bray, Upper critical dimension for the de Almeida-Thouless instability in spin glasses, J. Phys. C 16, L815 (1983).

[23] D. S. Fisher and H. Sompolinsky, Scaling in Spin-Glasses, Phys. Rev. Lett. 54, 1063 (1985). 
[24] S. F. Edwards and P. W. Anderson, Theory of spin glasses, J. Phys. F 5, 965 (1975).

[25] E. Pytte and J. Rudnick, Scaling, equation of state, and the instability of the spin-glass phase, Phys. Rev. B 19, 3603 (1979).

[26] A. J. Bray and M. A. Moore, Replica symmetry and massless modes in the Ising spin glass, J. Phys. C 12, 79 (1979).

[27] I. R. Pimentel, T. Temesvári, and C. De Dominicis, Spinglass transition in a magnetic field: A renormalization group study, Phys. Rev. B 65, 224420 (2002).

[28] G. Parisi and T. Temesvári, Replica symmetry breaking in and around six dimensions, Nucl. Phys. B858, 293 (2012).

[29] G. Kotliar, P. W. Anderson, and D. L. Stein, Onedimensional spin-glass model with long-range random interactions, Phys. Rev. B 27, 602 (1983).

[30] D. R. Bowman and K. Levin, Spin-glass theory in the Bethe approximation: Insights and problems, Phys. Rev. B 25, 3438 (1982).
[31] D. J. Thouless, Spin Glass on a Bethe Lattice, Phys. Rev. Lett. 56, 1082 (1986).

[32] G. Parisi, F. Ricci-Tersenghi, and T. Rizzo, Diluted meanfield spin-glass models at criticality, J. Stat. Mech. (2014) P04013.

[33] C. A. Doty and D. S. Fisher, Correlation functions of the Ising spin glass on the Bethe lattice, Phys. Rev. B 39, 12098 (1989).

[34] A. J. Bray and M. A. Moore, Non-analytic magnetic field dependence of the magnetisation in spin glasses, J. Phys. A 17, L613 (1984).

[35] J. Gertler and J. Machta, Absence of chaotic size dependence for spin glasses on hierarchical lattices, arXiv: 1711.10666.

[36] P. Charbonneau and S. Yaida, Nontrivial Critical Fixed Point for Replica-Symmetry-Breaking Transitions, Phys. Rev. Lett. 118, 215701 (2017). 